\title{
Crédito bancario y crecimiento económico en México
}

\author{
Bank credit and economic growtn in Mexico
}

\author{
José Luis Clavellina Miller*
}

\begin{abstract}
Resumen
El presente trabajo hace una breve descripción del proceso de extranjerización de la banca comercial en México, así como de las principales características actuales del mercado crediticio del país, entre éstas últimas sobresalen la escasez, la concentración y poder de mercado con que cuentan las instituciones bancarias que lo ofrecen, así como los altos costos que tienen que pagar los usuarios del mismo. Por el lado de la demanda se mencionan algunos de los elementos que limitan el acceso de las pequeñas y medianas empresas al crédito bancario, tales como un alto grado de informalidad y el crédito que reciben de proveedores. La segunda parte del trabajo se dedica a explorar la forma en que el crédito bancario apoya al crecimiento económico del país; si bien dicha relación es apoyada por diversos estudios teóricos y empíricos que buscan explicar las causas del crecimiento económico, para el caso de México se encuentra que, para el período de estudio, dicha relación no se cumple. Una de las posibles causas de lo anterior es que las instituciones financieras privadas no han privilegiado el crédito a los sectores productivos, sino que lo han canalizado al sector público (que presenta un bajo nivel de morosidad) y al consumo (al cual cargan mayores intereses y les es más rentable).
\end{abstract}

\footnotetext{
Palabras clave:

- Bancos

- Políticas de financiamiento

- Crecimiento económico
}

\begin{abstract}
This paper briefly describes the process in which foreign banks have increased their participation in the Mexican financial system; some of the main features of the credit market are also mentioned, such as scarcity, concentration and market power of the main participants, as well as high interest rates charged. On the demand side, some of the main elements that limit the access of small and medium enterprises to the private credit (that is, informality and informal credit) are also mentioned. The second part of the article explores the way banking credit supports economic growth. In spite of the fact that this relationship is found in many theoretical and empirical works, in the case of Mexico, it was not validated by this work. One of the possible causes of this result is that financial institutions in the country have not privileged the productive sector as the main destination of its credit. Commercial banks have preferred to provide credit to the government (due to its very low delinquency rates) and to consumption (because of the high interest rates they charge on it).
\end{abstract}

Clasificación JEL: G21, G32, 047

\section{Introducción}

Entre los distintos limitantes al crecimiento económico que enfrenta nuestro país, la disponibilidad de crédito productivo es uno de los más importantes. Tras la crisis financiera de 1994-1995 el crédito al sector privado en México se contrajo y no ha podido recuperarse.

En una economía en desarrollo, donde son pocas las empresas que tienen la posibilidad de acceder al financiamiento, o bien, a la colocación de títulos de deuda y acciones en los mercados de capitales, el crédito bancario se vuelve un elemento esencial en el apoyo a sus actividades productivas. Pese a ello, el crédito bancario en

\footnotetext{
* Doctor en Economía por la UNAM, ha trabajado temas relacionados con el financiamiento para el desarrollo y en torno a la relación entre desarrollo financiero y crecimiento económico. Actualmente es profesor de la División de Estudios Profesionales y de la División de Estudios de Posgrado de la Facultad de Economía de la UNAM, e investigador en la Dirección de Estudios Macroeconómicos del Centro de Estudios de las Finanzas Públicas de la Cámara de Diputados
} 
México es escaso y caro. Para tratar de analizar el por qué de estas características, se explorarán brevemente algunas de las condiciones de oferta y demanda en el mercado de crédito. Se describe también la alternativa que las empresas mexicanas han encontrado para hacerse de recursos cuando no son sujetas de crédito; es decir, se mencionan algunas de las ventajas y desventajas que el financiamiento de proveedores representa para la mayor parte de las empresas del país.

Posteriormente se intenta determinar si durante los últimos años el crédito ha sido funcional al crecimiento económico, así como analizar la relación causal que existe entre ambas variables.

Pese a que la reforma financiera se llevó a cabo en nuestro país al mismo tiempo que otras (como la liberalización comercial y el redimensionamiento del Estado) y que la medición del impacto que ésta ha tenido en el desempeño de nuestra economía no se puede aislar completamente, se realiza un ejercicio estadístico simple para intentar establecer si existe un parámetro que nos permita cuantificar la forma en que el crédito bancario apoya el crecimiento. El análisis estadístico se lleva a cabo utilizando información para el mercado crediticio en México, en el período que va de 1995 a 2012 . Es decir, se analiza el comportamiento del crédito bancario y su relación con el crecimiento económico en los años posteriores a la crisis económica de 1994-1995.

La teoría económica establece que la disponibilidad de financiamiento es una condición fundamental para el apoyo al crecimiento económico. El papel que juegan los intermediarios financieros es el de captar recursos de la sociedad para canalizarlos en forma de créditos a las empresas con proyectos más rentables, propiciando con ello un mayor crecimiento económico. Otra de las funciones de los intermediarios financieros es que permiten administrar riesgos, pues diversifican los plazos entre los recursos que captan y los que prestan, de tal forma que los agentes económicos que ahorran no quedan expuestos de forma directa a aquellos que utilizan los recursos financieros.

Los intermediarios financieros ofrecen, además, la ventaja de mantener cierta vigilancia entre sus acreditados; es decir, los intermediarios conocen el historial crediticio de sus clientes y el comportamiento que han tenido en sus cuentas, poseen información sobre la situación financiera de un gran número de agentes y orientan sus recursos a aquellos que les ofrecen mayor seguridad.

Así, el crecimiento económico a largo plazo depende, entre otras cosas, de la capacidad de aumentar las tasas de acumulación de capital, de la utilización de los activos de manera eficiente; lo cual se logra a través de una intermediación financiera que movilice el ahorro familiar y externo a la inversión empresarial, asegurando que los fondos se asignen de forma más productiva y diversifiquen el riesgo, proporcionando liquidez a las empresas (FitzGerald, 2007). 
Pese a las bondades que supone la existencia de instituciones financieras, es preciso indicar que, en ocasiones, la teoría económica convencional no tiene en cuenta la calidad de las mismas, ni los problemas relacionados con la información asimétrica y la competencia imperfecta que caracterizan al sector financiero y que pueden producir niveles de financiamiento inferiores al óptimo, así como una distribución ineficiente de recursos en detrimento del crecimiento económico (Stiglitz y Weiss, 198I).

Durante buena parte del siglo xx se consideró al sistema financiero de México como reprimido, pues hasta la década de los ochenta el gobierno establecía tasas de interés, encajes legales y cajones de crédito. Por ello, en las décadas de los ochenta y noventa se impulsaron reformas financieras que pretendían liberar al sistema, elevar el ahorro de la población y con ello, estar en posibilidades de otorgar una mayor cantidad de créditos.

Los defensores de la liberalización financiera argumentaban que ésta conducía a un mayor desarrollo financiero a través de una más alta disponibilidad de crédito, resultante de la abolición de los topes a las tasas de interés. Adicionalmente, la selección de proyectos de inversión por parte de las instituciones financieras ayudaría a elevar la productividad marginal del capital (McKinnon, 1973).

No obstante, enfoques más críticos argumentaban que la liberalización promovería una menor disponibilidad de fondos para el sector productivo, pues las mayores tasas de interés real redistribuirían el ahorro (tradicional) hacia nuevos activos financieros, dejando el ahorro total inalterado y reduciendo así la cantidad de fondos prestables para el sector productivo (FitzGerald, 2007).

\section{Antecedentes}

El sistema bancario en México es resultado de una compleja transformación en la que se encuentran procesos de desregulación, expansión, crisis, estatización, privatización y extranjerización. Así por ejemplo, en la década de los cuarenta existía una marcada división entre banca comercial e instituciones de inversión. En los cincuenta se obligaba a los bancos comerciales a mantener en el Banco de México depósitos proporcionales a las obligaciones que tenían por depósitos de reclamo inmediato; lo mismo que en los sesenta donde además, el financiamiento a la inversión tuvo gran participación estatal.

Uno de los acontecimientos fundamentales en la década de los setenta fue la creación de la banca múltiple (en 1974), la cual comenzó operaciones en 1976. Se pretendía que ésta asegurara financiamiento a mayores plazos para la industria; que avanzara en la asignación eficiente del crédito, homogeneizara el régimen de encaje 
legal, incrementara la oferta de productos financieros y mejorara el control y la vigilancia de las instituciones (Girón, et al., 2005).

Hacia finales de la década de los setenta la economía mexicana atravesó por una serie de desequilibrios como: un menor ritmo de crecimiento, acelerada inflación, elevados coeficientes de dolarización, imposibilidad de complementar el ahorro interno con flujos de capital externo; sobrevaluación cambiaria, un déficit comercial insostenible agravado por la caída en los precios del petróleo, alto déficit público y un importante incremento en las tasas de interés internacionales. Todos estos hechos causaron además importantes afectaciones a las finanzas de los bancos comerciales que culminaron con su estatización.

Cabe señalar que si bien se presentó un álgido debate en torno a si la nacionalización bancaria fue una medida adecuada o no, especialistas como Tello (1984) y Solís (1997) establecen que se trató de una determinación que irremediablemente se debía tomar debido a que un buen número de bancos se encontraban técnicamente quebrados.

En 1983 los bancos comerciales fueron trasformados en sociedades nacionales de crédito; más tarde, en 1985 con el objeto de racionalizar y optimizar sus operaciones se redujo su número. De 60 instituciones existentes en 1982 se revocaron II concesiones, se fusionaron 28 bancos en 12 sociedades y 17 más conservaron su denominación con lo que se contaba con 29 bancos, posteriormente, entre 1985 y 1988 se fusionaron II bancos más, totalizando así 18 sociedades nacionales de crédito (Solís, 1997).

A partir de 1988 el país inició un proceso de reforma económico-financiera. Paulatinamente se eliminaron los cajones selectivos de crédito y se liberalizaron las tasas de interés. Poco después, en mayo de 1990, el gobierno anunció la desincorporación bancaria y expidió las bases para dar inicio al proceso en septiembre del mismo año. Entre los objetivos de la reprivatización se encontraban: contar con un sistema financiero eficiente y competitivo; garantizar una participación diversificada y plural en el capital de los bancos; vincular la aptitud con la calidad moral de la administración de los mismos; asegurar que la banca fuera controlada por nacionales; lograr descentralización y arraigo regional en las instituciones; obtener un precio justo por ellos; conformar un sistema financiero balanceado y propiciar prácticas bancarias y financieras sanas (Solís, 1997).

Entre 1991 y 1992, se desincorporaron los 18 bancos que hasta ese momento pertenecían al gobierno. Para algunos especialistas, el mecanismo de privatización fue inapropiado pues benefició a casas de bolsa que, en algunos casos, eran propiedad de los antiguos administradores de la banca comercial. Además, los bancos recién privatizados aprovecharon la inadecuada regulación y supervisión para propi- 
ciar un excesivo aumento en el crédito (por encima de la dinámica real de la economía), a lo que se sumaron las precarias prácticas de valuación del riesgo y el rezago tecnológico en el sector bancario de esa época (de la Cruz et al., 20 I I).

Dos años más tarde, en 1994, se presentó una salida de capitales producto de desequilibrios monetarios y acontecimientos políticos que contaminaron el ambiente económico, lo que redujo considerablemente la liquidez del mercado. Lo anterior se agravó por la emisión de bonos gubernamentales a corto plazo denominados en dólares y por la libre convertibilidad, que ocasionaron una rápida reducción de las reservas internacionales y una crisis económica. Tanto la crisis como la devaluación de la moneda (en diciembre de 1994) incrementaron la cartera vencida bancaria. Además, la elevación de las tasas de interés y el mayor desempleo, exacerbaron el incumplimiento de las obligaciones de los deudores, causando graves problemas en el sector bancario y obligando al gobierno a adelantar la entrada del capital extranjero al sector con el fin de encontrar fuentes alternas de capitalización.

Si bien éste proceso de apertura incrementó la capitalización del sistema, también redujo el número de bancos, concentrando aún más el poder de mercado de las instituciones sobrevivientes. De los 18 bancos existentes en 1988 y derivado del proceso de adquisiciones y fusiones, sobreviven actualmente seis: Citicorp-Banamex, BBVA-Bancomer, HSBC, Scotiabank-Inverlat, Santander-Serfin y Banorte; de los cuales sólo uno (Banorte) tiene capital nacional mayoritario. Aunque en la actualidad existen poco más de 40 instituciones bancarias en el país, son éstas seis las que mantienen la mayor parte de los activos, la cartera y las utilidades del sistema en el país.

Entre los argumentos en favor de una mayor presencia de bancos extranjeros se encontraba que éstos aportarían nueva tecnología y técnicas administrativas, elevarían la productividad y la competencia del sector y se contaría con fuentes de acceso a capital internacional.También, se decía que serían una fuente de estabilidad ante turbulencias financieras internacionales y que los bancos centrales de los países de origen monitorearían la conducta de sus bancos apoyando a los reguladores locales. Otros estudios mostraban que la presencia de bancos extranjeros incrementaba la estabilidad de los sistemas locales. Crystal, Dages y Goldberg (200I) argumentaban que los bancos extranjeros poseían una mayor cantidad de reservas que los locales y que trataban con mayor firmeza el deterioro en la calidad de su cartera. Por otro lado, existían también, preocupaciones por la entrada de la banca extranjera. Una de las principales era que dado su tamaño, estos podían ser demasiado grandes para ser supervisados, y que sus intereses serían distintos a los del país; incluso que podían servir de conducto para grandes entradas y salidas de capital que distorsionarían el mercado, además de que sectores de bajos ingresos y pequeñas empresas quedarían sin atención por parte de éste tipo de intermediarios (Stallings, 2006). 


\section{Cuadrol}

Fusiones, Adquisiciones y Extranjerización de la Banca en México

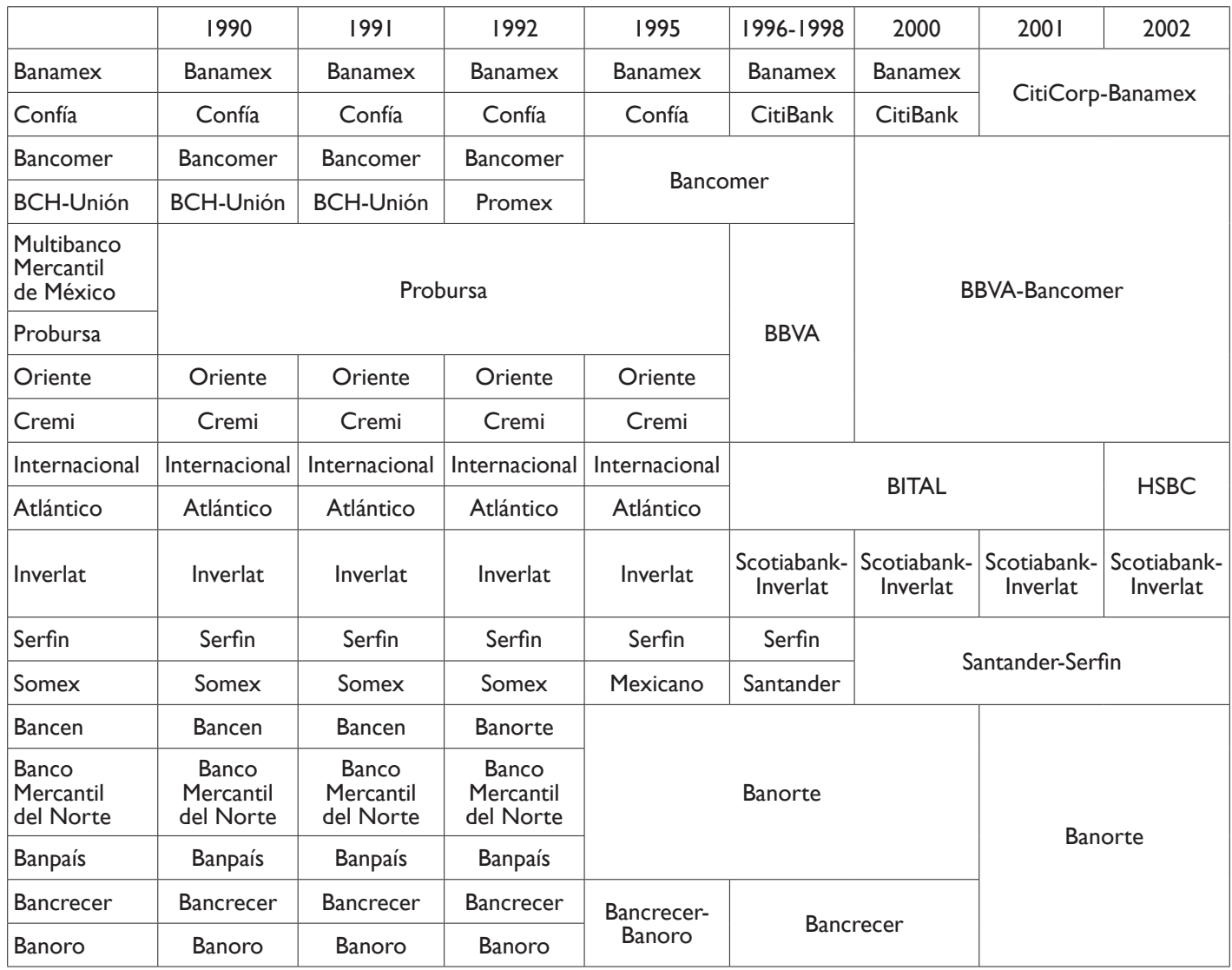

Fuente: elaboración propia con datos de Girón et al. (2005) y Centro de Estudios Espinoza Yglesias.

\section{Características del mercado crediticio en México}

\section{a) Escasez}

Como se mencionó anteriormente, debido a que los mercados de capitales en las economías emergentes no están ampliamente desarrollados, las instituciones bancarias juegan un papel muy importante en el financiamiento a las actividades productivas de las empresas pequeñas y medianas, que son las principales generadoras de empleo. Pese a la relevancia que tiene como fuente de financiamiento para las empresas, el crédito que otorga la banca mexicana como proporción del PIB es bajo. De acuerdo con datos del Banco Mundial (20II), dicha relación se ubicó en 26.1\%, mientras que 
para el caso de países como Japón, el mismo indicador fue de $169.75 \%$ y para otras economías en desarrollo como China y Brasil, los porcentajes fueron de 127.37 y $61.39 \%$, respectivamente.

\section{Gráfica I}

Crédito Interno al Sector Privado como porcentaje del PIB

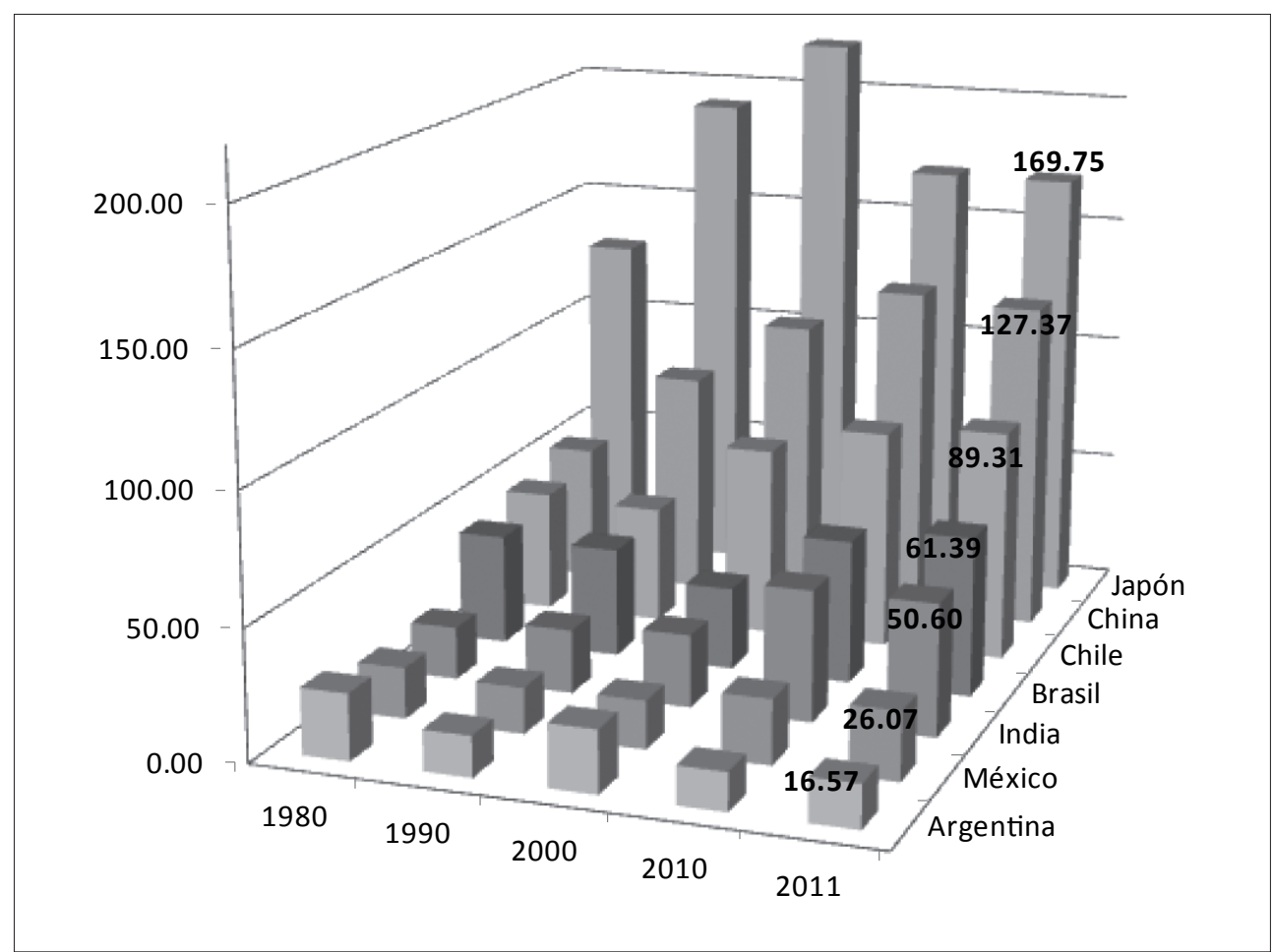

Fuente: Banco Mundial (20II).

\section{b) Concentración}

A la escasez de crédito se añade un factor, el reducido número de instituciones bancarias que lo ofrecen y que por ende gozan de una posición privilegiada en el mercado; esto es, en lo que se refiere a la fijación de tasas de interés y comisiones. A marzo de 2012, existían en el país 42 instituciones bancarias, pero sólo siete de ellas mantenían la mayor parte de los activos (79.0\%) y de la cartera de crédito (74.4\%); mientras que las 21 instituciones más pequeñas apenas tienen 3.9 y $3.7 \%$, respectivamente. 


\section{Cuadro 2}

Concentración Bancaria en México, marzo de 2012

\begin{tabular}{|ll|ll|}
\hline I & BBVA Bancomer & 22 & Barclays \\
2 & Banamex & 23 & Multiva \\
3 & Santander & 24 & CIBanco \\
4 & Banorte & 25 & Ve por Más \\
5 & HSBC & 26 & American Express \\
6 & Inbursa & 27 & Bancoppel \\
7 & Scotiabank & 28 & Bansí \\
8 & Deutsche Bank & 29 & Compartamos \\
9 & Banco del Bajío & 30 & Banco Ahorro Famsa \\
I0 & Bank of America & 31 & Inter Banco \\
II & Afirme & 32 & Bank of Tokyo-Mitsubishi \\
I2 & Ixe & 33 & Banco Base **/ \\
I3 & Interacciones & 34 & Actinver \\
I4 & Banco Azteca & 35 & UBS \\
I5 & Banregio & 36 & Banco Wal-Mart \\
I6 & Banco Credit Suisse & 37 & ABC Capital \\
I7 & ING & 38 & Volkswagen Bank \\
I8 & Invex & 39 & Autofin \\
I9 & Banca Mifel & 40 & The Royal Bank of Scotland \\
20 & JP Morgan & 41 & The Bank of New York \\
21 & Monex & 42 & Banco Fácil \\
\hline
\end{tabular}

Fuente: CNBV.

Claramente, las características en que opera el mercado bancario en el país son oligopólicas. Si se compara la participación de los bancos en el mercado con su nivel de rentabilidad, se puede apreciar que las instituciones más grandes son las mismas donde esta última es mayor. 


\section{Gráfica 2}

Participación en el Mercado y Rendimiento sobre Capital de la Banca Comercial en México, marzo de 2012

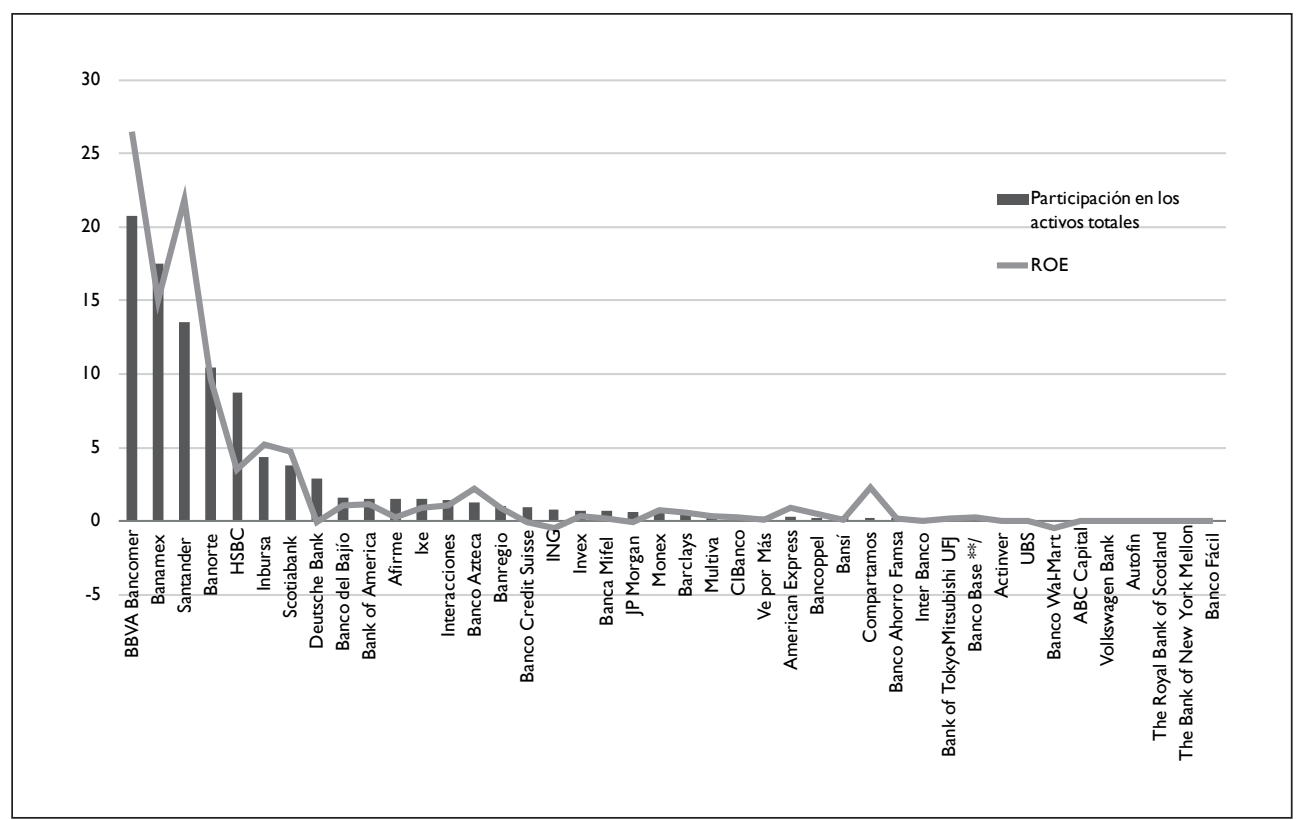

Fuente: CNBV.

Si bien lo anterior puede indicar la existencia de economías de escala en el negocio bancario, es decir, que se obtienen mayores rendimientos conforme el tamaño de los bancos crece; es también evidencia de que los bancos grandes al maximizar sus beneficios obtienen rentas extraordinarias por su participación en el mercado.

Según el Instituto Mexicano para la Competitividad (IMCO, 20I2), la estructura del sistema bancario mexicano no funciona precisamente como un oligopolio, sino que se trata de un modelo de competencia monopolística, donde las instituciones intentan diferenciar sus productos, resultándole a los consumidores difícil diferenciar entre las distintas ofertas del mercado; no obstante, el citado instituto también habla de un modelo de comportamiento de competencia imperfecta.

\section{c) Distribución}

En lo que se refiere a la forma en que se distribuye el crédito de la banca comercial en el país, resulta interesante observar que durante los últimos años, prácticamente todas las ramas productivas han visto disminuida su participación dentro de la carte- 
ra total. Comparando diciembre de 1995 con junio de 2012, se tiene que el crédito orientado al sector agropecuario pasó de 5.2 a $1.6 \%$; el del sector industrial de 24.7 a $20.0 \%$; el del sector servicios, de 31.3 a $22.0 \%$; y el correspondiente al crédito a la vivienda de 21.4 a 16.7 por ciento.

Por otro lado, entre los rubros que vieron incrementada su participación se encuentra el crédito al consumo, que de 5.3 a $21.2 \%$; mientras que el orientado al sector público pasó de 4.0 a $13.2 \%$, es decir, es posible observar que la banca privada ha privilegiado el crédito a sectores no productivos, aunque quizá más rentables como en el caso del crédito al consumo, o más seguros como en el crédito al sector público. Lo anterior puede ocasionar que las mediciones estadísticas sobre la relación entre crédito y crecimiento pueda tener resultados contrarios a los señalados por la teoría.

\section{Cuadro 3}

Distribución del crédito otorgado por la banca comercial según actividad de prestatarios (porcentajes)

\begin{tabular}{|l|r|r|r|r|r|}
\hline \multicolumn{1}{|c|}{ Título } & Dic 1995 & Dic 2000 & Dic 2005 & Dic 2010 & Jun 2012 \\
\hline Total (I a X) & 100.0 & 100.0 & 100.0 & 100.0 & 100.0 \\
\hline $\begin{array}{l}\text { Sector privado del país (I a VI) - } \\
\text { Empresas y personas físicas }\end{array}$ & 90.7 & 73.8 & 75.0 & 81.1 & 81.6 \\
\hline I. Sector agro., silvícola y pesquero & 5.2 & 3.8 & 1.8 & 1.6 & 1.64 \\
\hline II. Sector industrial & 24.7 & 19.6 & 13.9 & 21.0 & 20.0 \\
\hline III.Sector servicios y otras actividades & 31.3 & 23.4 & 20.4 & 21.5 & 22.0 \\
\hline IV. Crédito a la vivienda & 21.4 & 21.1 & 15.5 & 17.9 & 16.7 \\
\hline V. Créditos al consumo & 5.3 & 4.6 & 23.4 & 19.1 & 21.2 \\
\hline VI. Renglón de ajuste estadístico & 2.9 & 1.3 & 0.0 & 0.0 & 0.0 \\
\hline VII. Sector financiero del país & 3.7 & 2.9 & 5.4 & 2.5 & 3.4 \\
\hline VIII. Sector público & 4.0 & 5.2 & 9.0 & 13.6 & 13.2 \\
\hline Ix. Oros & & 17.7 & 9.9 & 0.5 & 0.3 \\
\hline X. Sector externo & 1.5 & 0.5 & 0.7 & 2.2 & 1.5 \\
\hline XI. Crédito intrabancario & 2.7 & 6.2 & 0.1 & 0.1 & 0.1 \\
\hline
\end{tabular}

Fuente: elaboración propia con datos de Banxico.

Así, tras la crisis financiera de 1994-1995, la reestructuración de los bancos y su cartera de créditos no privilegió al aparato productivo, sino que se orientó a sectores más rentables y seguros (consumo y gobierno), ocasionando con ello un deterioro en la posición financiera de las familias, lo que posiblemente limita también la cantidad de recursos disponibles para las actividades productivas. 


\section{d) Precios}

La concentración de mercado y la falta de competencia se ven reflejados en los precios que los clientes pagan por el uso de los servicios y productos que la banca ofrece, así como en los rendimientos que reciben por sus ahorros. El diferencial entre tasas de interés activas y pasivas de la banca comercial es amplio, por ejemplo, la tasa de interés promedio efectiva en tarjetas de crédito para clientes no totaleros (aquellos que no cubren la totalidad de su adeudo al llegar su fecha de corte) se ubicó en $\mathbf{2 9 . 8 \%}$ en febrero del presente año, mientras que para ese mismo mes el rendimiento del pagaré con rendimiento liquidable al vencimiento (28 días) fue de apenas I.26 por ciento.

\section{Gráfica 3}

Tasas de Interés Activas y Pasivas

(por ciento anual)

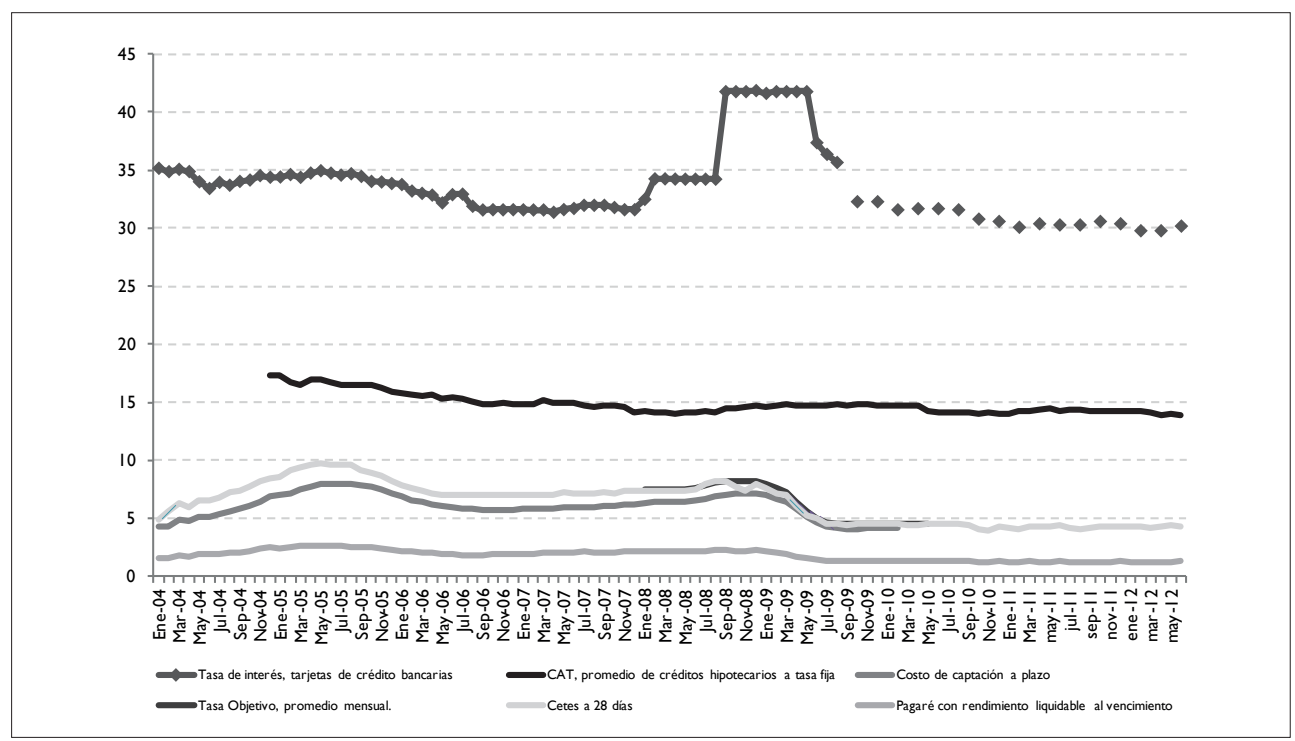

Nota: a partir de octubre de 2009 para el caso de las tarjetas de crédito se refire a la tasa efectiva promedio ponderada para clientes no totaleros.

Fuente: BANXICO.

\section{e) Otras condiciones de oferta}

Teóricamente, las instituciones bancarias al ser un elemento fundamental en el financiamiento de las actividades productivas deberían ocupar un papel predominante en el ciclo de negocios del país; no obstante, en México el proceso de reestructuración 
bancaria parece haber reducido el contacto y las relaciones de largo plazo entre bancos y sus clientes; esto es, la nacionalización, privatización, extranjerización y consolidación han impedido que las relaciones entre clientes y bancos perduren, pues dichos procesos han estado acompañados de la apertura y cierre de sucursales, así como de una gran movilidad laboral para los empleados bancarios.

Además, la decisión de los bancos de reducir la disponibilidad de crédito puede hallarse en que para la canalización del mismo se basan en aspectos distintos al precio, como lo puede ser el riesgo de no reembolso o la asimetría de la información (Stiglitz y Weiss, I 98I).

\section{f) Características de los demandantes de crédito}

Desde la perspectiva de la banca comercial, los altos intereses que caracterizan al crédito que se otorga a las Pequeñas y Medianas Empresas (PYMES) obedece, entre otras cosas a su alta mortalidad; a la insuficiencia en la información sobre estas empresas; asimetrías de información y selección adversa; riesgo moral; dependencia de las garantías en la obtención de créditos; un orden jurídico deficiente y una creciente informalidad en la economía (Lecuona, 2009).

Los mayores riesgos que enfrentan las PYMES para sobrevivir explican las mayores tasas de interés que tienen que pagar por los créditos que requieren. Por otro lado, es común que las PYMES cuenten con una estructura poco profesional, con un escaso uso de técnicas contables y financieras y donde las finanzas personales del propietario se mezclan con las de su negocio; lo que provoca, en varias ocasiones, daño moral cuando el empresario no destina los préstamos para incrementar la capacidad productiva de su negocio, sino que lo desvía a otros gastos. Lo anterior aunado a la gran informalidad con que operan este tipo de unidades y la escasa facturación, ocasionan que la información requerida para el otorgamiento de un crédito sea escasa y poco confiable.

Otra consecuencia de lo anterior es que ante la inexistencia de información contable y la ausencia de historiales crediticios, los bancos requieran de más documentación y garantías para otorgar un préstamo, incrementando con ello los costos de transacción. La escasez de información alimenta también el problema de selección adversa; no obstante, también es justo señalar que en la mayor parte de los países en desarrollo, las instituciones financieras carecen de empleados con la preparación suficiente para evaluar proyectos de inversión de forma adecuada (FitzGerald, 2007).

En el mismo sentido, el IMco (2012) establece que la legislación mercantil del país eleva los riesgos para hacer negocios, en especial para los intermediarios finan- 
cieros, pues los acreedores se ven afectados de forma importante al momento de demandar el incumplimiento de un contrato, ya que durante ese tiempo los créditos no pagan ni generan intereses. Otro de los problemas relacionados con la debilidad jurídica se encuentra en la falta de especialización de los tribunales civiles, los cuales atienden todo tipo de asuntos (familiares, patrimoniales y mercantiles), lo que ocasiona que los costos relacionados con el otorgamiento de créditos aumenten pues tienen que cubrir el riesgo jurídico.

\section{g) Crédito de proveedores}

Ante la escasez de créditos y la imposibilidad de cubrir los requisitos de la banca comercial para acceder a su financiamiento, las empresas mexicanas han recurrido al financiamiento de proveedores para continuar operando. De acuerdo con datos de la encuesta trimestral de financiamiento a empresas elaborada por Banco de México (Banxico), al segundo trimestre de 2012, en promedio, los proveedores representaron la principal fuente de recursos para $78.4 \%$ de las empresas encuestadas, mientras que la banca comercial y de desarrollo lo fueron para 39.3 y $5.6 \%$, respectivamente.

\section{Cuadro 4}

Resultados de la Encuesta de Evaluación Coyuntural del Mercado Crediticio (porcentajes)

\begin{tabular}{|c|c|c|c|c|c|c|}
\hline & & $\begin{array}{c}\text { Jul-Sep } \\
2010\end{array}$ & $\begin{array}{l}\text { Jul-Sep } \\
2011\end{array}$ & $\begin{array}{c}\text { Ene-Mar } \\
2012\end{array}$ & $\begin{array}{l}\text { Abr-Jun } \\
2012\end{array}$ \\
\hline \multirow{9}{*}{ 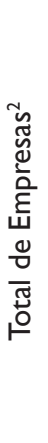 } & \multirow{9}{*}{ 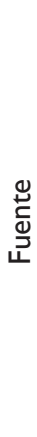 } & Empresas que obtuvieron algún tipo de financiamiento:' & 92.3 & 91.4 & 93.2 & 88.3 \\
\hline & & De proveedores & 82.7 & 84.9 & 82.9 & 78.4 \\
\hline & & De la banca comercial & 29.3 & 36.3 & 35.2 & 39.3 \\
\hline & & De la banca en el extranjero & 3.2 & 6.3 & 6.2 & 4.3 \\
\hline & & De empresas del grupo corporativo/oficina matriz & $N / E$ & 20.2 & 25.5 & 21 \\
\hline & & De empresas del grupo corporativo & 15.0 & $N / E$ & $\mathrm{~N} / \mathrm{E}$ & $N / E$ \\
\hline & & De la banca de desarrollo & 1.8 & 5.6 & 5.1 & 5.6 \\
\hline & & De la oficina matriz en el exterior & 4.2 & $N / E$ & $N / E$ & $N / E$ \\
\hline & & Mediante emisión de deuda & 2.5 & 3.0 & 3.4 & 2.5 \\
\hline
\end{tabular}

I Muestra con cobertura nacional de por lo menos 450 empresas. La respuesta es voluntaria y confidencial.

${ }^{2}$ La suma de los porcentajes puede ser superior a 100 ya que las empresas pueden elegir más de una opción de financiamiento.

N/E No estimado.

Fuente: Banxico. 
Las empresas que obtuvieron nuevos créditos bancarios durante el trimestre (29.8\%), manifestaron haber encontrado condiciones más accesibles en lo que se refiere a plazos y montos ofrecidos por las instituciones bancarias, mientras que las condiciones menos favorables se encontraron en el refinanciamiento de créditos y en los tiempos de resolución del mismo. En lo que se refiere a costos, las empresas encontraron condiciones favorables en el caso de tasas de interés y menos favorables en las comisiones y otros gastos asociados al crédito bancario. Mientras que para las empresas que no obtuvieron nuevos créditos bancarios, los factores limitantes se encontraron principalmente, la situación económica general (para 52.7\% de las empresas); las tasas de interés del mercado de crédito (46.4\%); las condiciones de acceso al crédito bancario (42.4\%) y las ventas y rentabilidad de la empresa (4I.6\%).

Si bien el financiamiento de proveedores como fuente alternativa de recursos ofrece la ventaja de tasas de interés muy bajas, también va en detrimento de los flujos de efectivo de las empresas que lo utilizan, el cual es un parámetro que las instituciones financieras tienen en cuenta al momento de analizar el posible otorgamiento de créditos empresariales, lo cual limita la capacidad del sistema financiero para impulsar el crecimiento económico (Mántey, 2007).

\section{Causalidad entre crédito y crecimiento económico}

La relación existente entre financiamiento y crecimiento económico, es una de las premisas teóricas que, desde principios del siglo xx, economistas como Schumpeter (1912) propusieron; Schumpeter establecía que las instituciones bancarias canalizaban el ahorro de la sociedad, vía créditos, a los proyectos más rentables e innovadores, promoviendo con ello tanto el desarrollo tecnológico como el crecimiento económico.

Posteriormente, en la década de los cincuenta, Gurley y Shaw (1955) establecían que las empresas podían elevar su nivel de eficiencia acudiendo a los mercados de deuda para financiar sus proyectos productivos. Más tarde, en los setenta, los estudios de Mckinnon (1973) y Shaw (1973), intentaban mostrar que en las economías en vías de desarrollo, la aplicación de topes a las tasas de interés pasivas y activas, los controles en el otorgamiento de crédito y en general la intervención gubernamental en el mercado financiero, reprimía su propio desarrollo, por lo que promovían medidas tendientes a liberalizar su sistema financiero.

Más adelante, trabajos como los de King y Levine (1993), intentaron demostrar empíricamente la existencia de una relación positiva entre el desarrollo del sistema financiero y el crecimiento. En el mismo sentido, Pagano (1993), encuentra que los 
intermediarios financieros eficientes incrementan la productividad del capital y por ende el crecimiento económico. Para este autor, los intermediarios recolectan información y reducen el riesgo de los inversionistas propiciando que estos decidan mantener activos cada vez menos líquidos pero a la vez más productivos.'

Honohan (2004) reconoce también un vínculo positivo entre financiamiento y crecimiento y asevera que éste último es indispensable para reducir los niveles de pobreza de una nación. Para él, existen dos canales por medio de los cuales la función financiera puede afectar el crecimiento económico: la acumulación de capital y la innovación tecnológica. En el primer caso, se afecta la acumulación de capital ya sea porque se altera la tasa de ahorro o por la reasignación de ahorros a través de las diferentes tecnologías productivas. Para éste autor, existen también otros factores que influyen en el funcionamiento financiero y el crecimiento económico, como lo son el sistema legal y las instituciones políticas.

Estos modelos establecen que el desarrollo financiero puede ayudar incluso a predecir el crecimiento porque suponen que éste se desarrolla con anticipación a la expansión del producto.Así, la existencia de diferencias entre el desarrollo financiero de distintas regiones, pueden alterar las tasas de crecimiento económico durante un amplio horizonte temporal (Beck, Levine y Loayza, 2000).

En contraposición a los hallazgos anteriores, autores como Thirlwall y Warman (1994) prueban la hipótesis de la liberalización financiera (que postula que un incremento en las tasas de interés reales provoca un crecimiento del ahorro, la inversión y el crecimiento económico), haciendo una importante distinción entre ahorro financiero y ahorro total. Ellos encuentran que, para el caso de México, de 1960 a 1990, el ahorro financiero tiene una relación positiva con la tasa de interés real; también que la inversión está positivamente relacionada con la oferta de crédito, pero que el efecto neto de las tasas de interés sobre la inversión es negativo, por lo que el círculo virtuoso del modelo de crecimiento económico que postulaba McKinnon (1973) no se validó para el caso mexicano. ${ }^{2}$

El modelo especificado por Warman y Thirwall (1994) mostraba también que la liberalización financiera se había traducido en volúmenes adicionales de ahorro financiero, que no se vinculaban de forma importante a la inversión en activos productivos vía canalización de créditos bancarios.

I En su modelo de crecimiento endógeno, Pagano (1993) encuentra además que el impacto de la tasa de interés real en el nivel de ahorro es ambiguo, el ahorro puede caer y reducir el crecimiento debido a que el desarrollo financiero incrementa la seguridad del mercado y por lo tanto los individuos pueden ahorrar menos por tener menos motivos precautorios.

2 Maxwell Fry (1995) había llegado a una conclusión similar algunos años antes cuando encontró que cualquier efecto favorable de la liberalización financiera y de las más altas tasas de interés en el crecimiento económico estaría dado por el incremento en la productividad de la inversión, más no por la adopción de tasas de interés reales positivas. 
También para el caso de México, De la Cruz (et al., 20 I l), encuentra que, el único tipo de crédito sectorial que impacta la actividad económica (medida a través del Intervención General de la Administración del Estado sectorial) es el otorgado al consumo y los servicios (aunque su efecto se focaliza sólo en ese sector), así como una relación bidireccional entre actividad económica y crédito. También encuentra una escasa relación entre la esfera financiera y la generación de valor agregado en el país.

En México, la evolución reciente del crecimiento PIB, la inversión y del crédito total muestra una tendencia similar en el período que va del cuarto trimestre de 2005 al primer trimestre de 2012.Conviene indicar que siguiendo a autores como FitzGerald (2007), en el presente análisis se utiliza a la variable de crédito real como medida de intermediación financiera. ${ }^{3}$

\section{Gráfica 4}

Crecimiento Real Anual del PIB, la Inversión y el Crédito 1995-2012/1

(porcentaje)

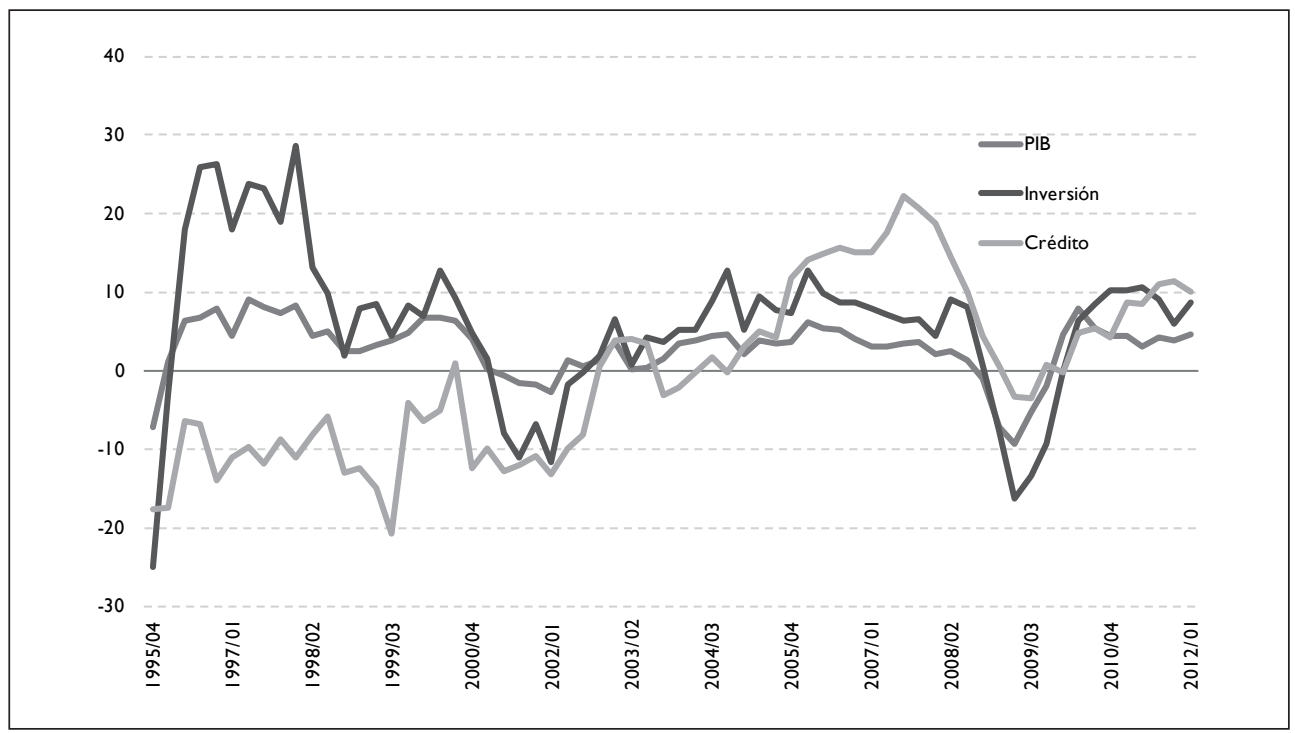

Fuente: Banxico e INEGI.

En el período referido, tanto el crecimiento del PIB real como el crecimiento de la inversión en México son variables estacionales, es decir, su orden de integración

3 FitzGerald (2007) considera que para medir el desarrollo financiero o el nivel de intermediación debe tenerse en cuenta el nivel de crédito, o bien, el crédito respecto del PIB y no tanto el ahorro financiero, medido éste último a través de algún agregado monetario al cual se le descuentan los billetes y monedas, pues considera que existen marcadas diferencias entre países en los estudios de corte transversal. 
es cero [l(0)], lo anterior fue resultado de la aplicación tanto de la prueba DickeyFuller como de la Phillips-Perron(aplicadas a las series sin intercepto ni tendencia). En lo que se refiere a la variable de crecimiento del crédito total ésta resultó ser de orden de integración uno, tras la aplicación de la prueba Dickey-Fuller; no obstante, fue de orden de integración cero en la Philips-Perron.

La regresión realizada con esas tres variables, usando como variable dependiente al crecimiento del PIB y como variables explicativas tanto a la inversión como el crédito total, arrojó una serie de errores que fueron estacionales, es decir, cuyo orden de integración fue cero por lo que se determinó que dichas variables estaban relacionadas.

\section{Cuadro 5}

Pruebas de raíz unitaria

\begin{tabular}{|c|c|c|c|c|c|c|}
\hline \multirow[b]{2}{*}{ Variable } & \multicolumn{3}{|c|}{$\begin{array}{l}\text { Dickey-Fuller aumentada en niveles, } \\
\text { sin intercepto ni tendencia }\end{array}$} & \multicolumn{3}{|c|}{$\begin{array}{l}\text { Philips-Perron en niveles, } \\
\text { sin intercepto ni tendencia }\end{array}$} \\
\hline & Estadístico - t & Prob. & $\begin{array}{l}\text { Orden } \\
\text { de integración }\end{array}$ & Estadístico - t & Prob. & $\begin{array}{c}\text { Orden } \\
\text { de integración }\end{array}$ \\
\hline $\begin{array}{l}\text { Crecimiento real del PIB } \\
\text { (CRECPIB) }\end{array}$ & 2.499 & 0.013 & $\mathrm{I}(0)$ & $-2.805 I$ & 0.0057 & $\mathrm{I}(0)$ \\
\hline $\begin{array}{l}\text { Crecimiento de la Inversión } \\
\text { (CRECINV) }\end{array}$ & -2.7948 & 0.0059 & $\mathrm{I}(0)$ & -3.288 & 0.0014 & $\mathrm{I}(0)$ \\
\hline $\begin{array}{l}\text { Crecimiento real del crédito total } \\
\text { (сстот) }\end{array}$ & -1.553 & 0.1123 & $\mathrm{I}(\mathrm{I})$ & -1.99 & 0.045 & $\mathrm{I}(0)$ \\
\hline Ut & -4.30021 & 0 & $\mathrm{I}(0)$ & -3.994 & 0.0001 & $\mathrm{I}(0)$ \\
\hline
\end{tabular}

En lo que se refiere a la causalidad entre las variables utilizadas, se tiene que las pruebas aplicadas al vector de corrección de errores, encontraron que tanto el crédito total como la inversión ayudan a explicar el comportamiento del PIB y que éste último tiene influencia significativa en la determinación de la inversión.

\section{Cuadro 10}

Posteriormente, en lo que se refiere al impacto que cada una de las variables tiene en el crecimiento del PIB, los resultados muestran al crecimiento de la inversión como un determinante estadísticamente significativo en el crecimiento del producto (con un coeficiente de 0.31 y un estadístico t de 16.56 ); mientras que el impacto que tiene el crédito en la determinación del producto resultó ser negativo, aunque 
muy pequeño y estadísticamente no significativo (el coeficiente fue $-0.00 \mathrm{II}$ y el estadístico t de -0.067).

\section{Cuadro 6}

VEC Causalidad de Granger /Block Exogeneity Wald Tests

Período : 1995Q4 20I2QI / 63 observaciones

Variable dependiente: Crecimiento real del crédito total

\begin{tabular}{|c|c|c|c|}
\hline Excluded & Chi-sq & df & Prob. \\
\hline Crecimiento del PIB real & 3.235470 & 2 & 0.1983 \\
\hline Crecimiento de la inversión & I.I6040I & 2 & 0.5598 \\
\hline \multicolumn{4}{|c|}{ Variable dependiente: Crecimiento del PIB real } \\
\hline Excluded & Chi-sq & df & Prob. \\
\hline D(сСтот) & 8.089375 & 2 & 0.0175 \\
\hline D(CRECINV) & 7.444571 & 2 & 0.0242 \\
\hline \multicolumn{4}{|c|}{ Variable: Crecimiento de la Inversión } \\
\hline Excluded & Chi-sq & df & Prob. \\
\hline D(сстот) & 1.968389 & 2 & 0.3737 \\
\hline $\mathrm{D}$ (CRECPIB) & 5.338001 & 2 & 0.0693 \\
\hline
\end{tabular}

Lo anterior es un indicio de que el postulado teórico que señala que el crédito al sector privado impulsa el crecimiento económico no se corrobora para el caso de la economía mexicana. Esto es, si bien las pruebas de causalidad indican que el crédito total tiene influencia en la determinación del producto, dicha influencia parece ser muy pequeña y negativa.

La ausencia de dicha relación, corroborada por distintos trabajos teóricos y empíricos como los referidos anteriormente, pero no encontrada en el presente análisis para el caso de nuestra economía puede deberse a distintas causas, entre las que destaca que la teoría da por sentada la alta calidad y eficiencia del sector financiero. Como se mencionó anteriormente, una baja calidad en la intermediación financiera puede ocasionar que se obtengan resultados no óptimos y que de hecho afecten el desempeño económico.

Así la calidad y eficiencia del sector bancario en México parecen explicar los resultados obtenidos por el presente trabajo, esto es, la falta de competencia en el sector, los altos intereses que se tienen que pagar por el crédito; la preferencia de los bancos por orientar sus recursos a actividades improductivas como el consumo (que les es más rentable) y al sector público (cuya morosidad es muy baja), aunado a lo pequeño que como proporción de la economía resulta el crédito otorgado por la 
banca, dan como resultado una disociación entre crédito y crecimiento económico para el caso de la economía mexicana.

Otro de los factores que puede ayudar a explicar el por qué de la disociación entre crédito y crecimiento económico para el caso de la economía mexicana se encuentra en que tomando en cuenta un horizonte de tiempo más amplio, y comparando la evolución del PIB con el crédito al sector privado como proporción del mismo, se puede apreciar que cada episodio de crisis está asociado a un distanciamiento entre ambas variables, situación que se hace más evidente tras la crisis de 1994-1995 (ver siguiente gráfica).

\section{Gráfica I 2}

Crédito interno al sector privado como proporción del PIB y Crecimiento real del PIB, I960-20II (porcentaje)

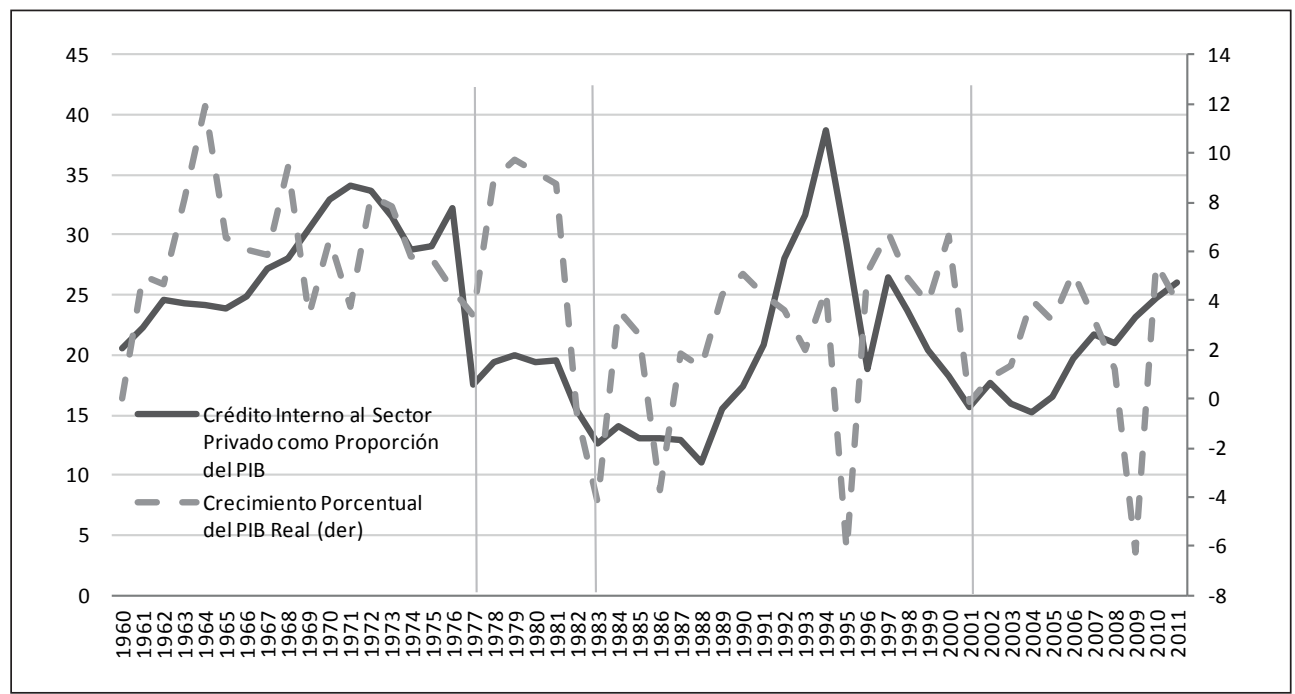

Fuente: Banco Mundial.

\section{Conclusiones y propuestas}

En México, los procesos de nacionalización bancaria de los ochenta, reprivatización y extranjerización de la banca en los noventa y 2000, no han sido capaces de alterar la estructura oligopólica del sector bancario, y tampoco han servido para elevar la competencia en el sector, reducir los costos del financiamiento y acercar los servicios a capas más amplias de la población. La escasez de crédito continúa siendo una de las principales limitantes al crecimiento económico del país. Respecto del tamaño de la economía, el financiamiento que otorga la banca privada en México es 
acotado y las condiciones en que lo hace se mantienen como un obstáculo para que las empresas obtengan recursos para detonar sus proyectos productivos.

Para promover el otorgamiento de crédito productivo en el país hace falta elevar la competencia en el sector bancario no sólo a través de políticas que castiguen las prácticas monopólicas, que son difíciles de evidenciar, sino a través de una mayor participación de la banca de desarrollo, la cual no debe priorizar únicamente su función de segundo piso sino aprovechar la infraestructura con la que cuenta en todo el país para promover el otorgamiento de créditos a los distintos sectores productivos del país. El número de sucursales con las que cuenta Bansefi, por ejemplo, es similar al que tiene un banco comercial como Scotiabank.

Ante la falta de financiamiento, las empresas han encontrado en el financiamiento de proveedores una forma para continuar operando en el mercado. No obstante, este tipo de financiamiento aunque representa ventajas en lo que se refiere a plazos y costos, no permite que se conformen las características necesarias para impulsar posteriormente el crédito bancario, pues reducen el flujo de efectivo de las empresas. Además se trata de un financiamiento que no promueve la inversión, sino que se destina a capital de trabajo.

La crisis de 1994-1995 ocasionó una reestructuración del sector bancario y de la cartera de crédito en el país. La necesidad de capitalizar los bancos obligó a abrirlos a la inversión extranjera. En la actualidad aunque los bancos se muestran financieramente sólidos y rentables, no otorgan el financiamiento que las empresas y familias requieren a costos accesibles. Una vez más, la promoción de la competencia en el sector requiere de una participación más activa de la banca de desarrollo, en el proceso la utilización de la infraestructura y sucursales de cajas de ahorro, cooperativas y microfinancieras, así como de corresponsales, sería fundamental.

Por otro lado, teórica y empíricamente diversos estudios han revelado la existencia de una relación positiva entre desarrollo financiero y crecimiento económico, no obstante dicha relación, no es una garantía; pues depende, entre otras cosas, de la eficiencia y calidad del sector financiero, del grado de competencia, de la participación del sector público en la cartera total de crédito bancario y de que los recursos financieros se orienten a los sectores productivos del país.

Al igual que otros estudios que se han realizado sobre el tema de análisis, el presente trabajo no encontró evidencia suficiente para asegurar que el proceso de liberalización financiera y el otorgamiento de crédito en la forma en que lo hace la banca comercial en la actualidad, abonen en un mayor crecimiento económico. Una posible explicación de lo anterior se encuentra en el hecho de que tras la crisis de 1994-I 995 se ha privilegiado el crédito al sector público y al consumo, esto último reduce el ahorro familiar agregado (la diferencia entre los activos y los pasivos fami- 
liares) y por ende la disponibilidad de recursos financieros. Así, ante los resultados encontrados por el presente trabajo, se hace necesaria la reconsideración del papel que juega no sólo la banca privada en el crecimiento económico del país, sino el que puede llegar a desempeñar la banca de desarrollo en el apoyo a los sectores productivos, y en la promoción de la competencia y acceso a servicios financieros de capas más amplias de la población; es decir, la intervención pública seguirá siendo necesaria en la corrección de las fallas del mercado presentes en el sistema financiero nacional.

Lo anterior debe ir de la mano del diseño de una política financiera que coordine y utilice tanto a la banca de desarrollo como a la banca comercial en el financiamiento al desarrollo del país, a través de la elaboración de instrumentos específicos para el financiamiento de proyectos productivos de sectores definidos y prioritarios.

Esto requiere que, de forma efectiva, de la alineación de los programas de gobierno (tanto el Programa Nacional de Desarrollo como el Programa Nacional de Financiamiento al Desarrollo) con los objetivos de los distintos bancos de desarrollo, y hacer partícipe a la Banca Comercial de los distintos fondos públicos y garantías para apoyar el otorgamiento de crédito a los distintos sectores productivos del país. Es decir, a fin de alcanzar las metas en materia de financiamiento y crecimiento deben coordinarse y complementarse los esfuerzos de los bancos, fideicomisos y fondos de fomento públicos con la infraestructura y alcance con que cuentan los bancos privados.

Resultaría indispensable además que el sector de ahorro y crédito popular y de cooperativas de ahorro y préstamo se consolide, con el objeto de que al igual que la banca comercial, sea partícipe de la estrategia de financiamiento al desarrollo y permita además, atender a un mayor número de personas en zonas alejadas de los centros urbanos.

El diseño de una política de financiamiento debe ir acompañado también de la asistencia técnica necesaria para que se superen las limitaciones que las empresas mexicanas tienen al momento de solicitar crédito a instituciones privadas, es decir, además de cuestiones productivas sería necesario atender cuestiones contables, administrativas y de capacitación empresarial (especialmente en el caso de las MIPYMES).

Lo anterior debe estar encaminado a la creación de sujetos de crédito, pues no debe olvidarse que el financiamiento otorgado por la banca de desarrollo se encuentra dentro del marco de una política pública, y que ésta busca atender un problema específico para un sector determinado de la población, por lo que su aplicación no puede ser permanente para los mismos agentes, es decir, si la aplicación de dicha política es exitosa, una vez terminados los plazos correspondientes, la po- 


\section{blación atendida debería ser capaz de acceder a fuentes de financiamiento privadas (a plazos y costos adecuados) para atender sus necesidades de recursos.}

\section{Bibliografía}

American Chamber/México, IMCO (2012), Crecimiento y profundización del sector financiero en México.

Banxico 1989, Informe Anual.

Beck, Thorsten, AsliDemirguc-Kunt y Ross Levine (2004), Finance, inequality and poverty: Crosscountry evidence. NBER working paper no. 10979, National Bureau of Economic Research, Cambridge, MA.

Crysral, J., Dages, G.,y Goldberg, L."Does foreign ownership contribute to sounder banks in emerging markets?" en Litan, E., et al., Open Doors: foreign participation in financial systems in developing countries, Brookings Institution Press, $200 \mathrm{I}$.

De la Cruz, J.L., y Alcántara, J.A., Crecimiento económico y el crédito bancario: un análisis de causalidad para México, ITESM, 20II.

FitzGerald Valpy (2007), Desarrollo Financiero y Crecimiento Económico: una visión Crítica, en Principios núm. /2007 pp. 5-28.

Girón, Alicia y Levy Noemi, 2005, México: Los bancos que perdimos. De la desregulación a la extranjerización del sistema financiero, IIEc, UNAM.

Gurley, J. and Eduard S. Shaw, 1955, "financial aspects of economic development" American Economic Review, 45 (4, September) 5I5-538.

Hodgman, D. (1960), Credit risk and credit rationing, en American Economic Review, vol. 69, núm. 6, pp. 850-872.

Honohan, P., Financial development, growth and poverty: how close are the links?, Policy research working paper series 3364, World Bank, 2004.

King, R.G. y Levine, R. (1993a), Finance and growth: Schumpeter might be right, Quarterly Journal of Economics 108 (3).

Lecuona Valenzuela Ramón, “El financiamiento a las PYMES en México. La experiencia reciente”, en EconomiaUnam vol. 6 núm. 17. México, 2009.

Levine, R., N. Loayza y T. Beck (2000), Financial intermediation and growth: Causality and causes, Journal of MonetaryEconomics 46 (I), pp. 3I-77.

Mántey, G. (2007), Política bancaria para el crecimiento con estabilidad, en Calva, J. (comp), Financiamiento del crecimiento económico, UnAM/Porrúa/Cámara de Diputados.

McKinnon, R. (1973), Money and Capital in Economic Development, Brookings Institutions, Washington $D C$. 
Peek, J., y Rosengren E., "implications of the globalization of the banking sector: the Latin American experience", New England Economic Review, Federal Reserve Bank of Boston, septiembre-octubre 2000.

Schumpeter Joseph, The Theory of Economic Development, Leipzig 1912. Harvard University press 1934.

Shaw E. 1973, Financial Deepening in Economic Development, NY. Oxford University Press.

Solís, Leopoldo, Evolución del sistema financiero mexicano hacia los umbrales del siglo XXI, Primera Edición, México, 1997.

Stallings, B. Financiamiento para el desarrollo. América Latina desde una perspectiva comparada, CEPAL, julio de 2006.

Stiglitz, J.y Weiss, A. (198I), Credit rationing in markets with imperfect information, American Economic Review 7 I (3), pp. 393-4I0.

Tello, M. Carlos, La nacionalización de la banca en México, Siglo Xxı, México, 1984.

Warman, F. y Thirlwall, A. P. (1994), Interest rate, savings, investment and growth in Mexico, 1960-1990: Tests of financialliberalization hypothesis, Journal of Development Studies 30 (3), pp. 629-649. 